\title{
DISEÑO DE UN PROTOTIPO PARA EL MANEJO DE DATOS EN EL MUSEO DE LA UNIVERSIDAD DE LA SALLE
}

\section{Prototype design for data management in the museum of the La Salle University}

\author{
Triana, Yesid'; Rodríguez, Diana²; Jaime, Yamile ${ }^{3}$ \\ Universidad de La Salle, Grupo de Investigación GIII
}

\section{Resumen}

Los Sistemas de Información permiten a las organizaciones de cualquier nivel contar con un activo organizado para la toma de decisiones estratégicas y administrativas que redundan en su beneficio y el del entorno. En el caso del Museo de la Universidad de La Salle, este alberga ocho colecciones biológicas; los datos de estas colecciones se registran actualmente en varios archivos en Excel, lo que no facilita su mantenimiento. El cuerpo de investigadores del Museo manifiesta que esta no es la manera más adecuada para trabajar con datos que requieren estar interrelacionados, esto significa que actualmente es necesario un arduo trabajo a la hora de buscar información para respaldar su trabajo.

En razón a lo anterior, el Museo requiere desarrollar un proyecto para implementar un sistema de información soportado en una base de datos relacional, que tenga la capacidad de almacenar los datos de sus colecciones y proveer información de manera organizada y eficaz. Para responder a esta necesidad, se plantearon cuatro fases, que corresponden a: (1) Análisis y diagnóstico de los archivos existentes, (2) Propuesta de diseño para la base de datos mediante un modelo entidad relación, (3) Desarrollo del diseño e implementación del sistema de reportes y consultas (4) Análisis del nuevo sistema mediante el desarrollo de pruebas con los actores del Museo, verificando la optimización de la información.

Se espera que, una vez desarrollado el sistema de información, este permita a los investigadores encontrar los datos requeridos de manera oportuna, facilitando además su mantenimiento al momento de realizar las actualizaciones exigidas por la normatividad que rige los museos

${ }^{1}$ Correo electrónico correspondiente: ytriana46@unisalle.edu.co

${ }^{2}$ Correo electrónico correspondiente: dianadrodriguez51@unisalle.edu.co

${ }^{3}$ Correo electrónico correspondiente: yajaime@unisalle.edu.co 
Palabras claves: Sistemas de información, Base de datos, Colecciones biológicas, Museo de La Salle.

\section{INTRODUCCIÓN}

La misión del Museo es: "contribuir a la recuperación y construcción de identidades (nacional, institucional, personal), hacer memoria de la tradición Lasallista, conservar y preservar por medio de sus colecciones, y proyectos de investigación y divulgación, el patrimonio natural y cultural convirtiéndose en una ventana interpretativa y en agente de cambio social a través de la apropiación social del conocimiento" [1] Lo que Esta investigación en sintonía con los objetivos de desarrollo sostenibles: (4) de una educación con calidad, (14) Conservar y utilizar sosteniblemente los océanos, los mares y los recursos marinos y (15) Gestionar sosteniblemente los bosques, luchar contra la desertificación, detener e invertir la degradación de las tierras, detener la pérdida de biodiversidad.

El Museo de la Universidad de La Salle es un agente de cambio social y desarrollo, inspirado en los principios del Código Profesional Deontología del ICOM [2], así como un promotor de los valores asociados al estudio de la diversidad biológica y cultural de Colombia. Se auto define como un escenario donde el encuentro de saberes y el ejercicio interdisciplinario se convierten en la dinámica propia de su accionar. Su objetivo principal es fomentar la investigación sobre el patrimonio que custodia y reflexionar sobre su quehacer pedagógico y museológico a través del patrimonio que conserva y preserva en sus colecciones. Como centro cultural y de apropiación social de conocimiento promueve actividades para el encuentro entre la ciencia y la comunidad que beneficia, mediante la difusión de la memoria biológica que alberga [3].

Las diversas colecciones con las que cuenta resguardan algunos de los especímenes más antiguas en el país, y otras de estas piezas incluso datan de periodos en los cuales el Museo de la Universidad de La Salle no se había constituido. Entre sus ejemplares se encuentran, en algunos casos, registros únicos de las especies que alguna vez vivieron en las proximidades de Bogotá, Cúcuta, Pamplona, Medellín, Villavicencio, Leticia, Florencia y que probablemente, ya estén extintas localmente Dada la antigüedad de estos ejemplares y la calidad de los mismos, se generaron grandes aportes al estudio del ecosistema colombiano y el Museo se destaca como un foco importante para la exhibición, restauración, conservación e investigación del patrimonio cultural, social y educativo para la transmisión de todo un legado cultural. [4]. 
Actualmente, el Museo se ha visto en la necesidad de clasificar en ocho colecciones sus especímenes, estas son: vertebrados, invertebrados, colecciones con fines científicos, depósito de ejemplares y donación de objetos, centro de documentación, exposiciones permanentes, exposiciones temporales y herbario. Cada una de ellas tienen específicamente su organización de acuerdo a sus respectivas características. Inicialmente, el Museo contaba con un software para el manejo de bases de datos biológicas, Specify, [5], Sin embargo, según los científicos del Museo, este software se volvió obsoleto ya que se debían hacer actualizaciones diarias para mantener al tanto la información de manera simultánea con respecto a las diferentes actividades de seguimiento de transacción de especímenes, enlaces de imágenes para los registros que se llevan a cabo en el Museo y demás procesos que se desarrollan allí [4]. Por lo tanto, los registros de las colecciones se empezaron a llevar en archivos de Excel, sin que esto representara una solución eficaz.

En cuanto a sistemas de información específicos para colecciones museológicas, a nivel internacional, se encuentran entre otros, el sistema Netx [6] plataforma líder en la gestión de activos digitales utilizado en museos como el Metropolitano de Arte de Nueva York (MET), el Museo de Brooklyn, y la Galería Nacional de Arte de Washington DC. Por otra parte, está el sistema Argus de Lucidea que brinda una plataforma web configurable, que permite la entrega de servicios de acuerdo a las necesidades de los usuarios [7]. También se encuentra, Altru, esta solución hace seguimiento asociado a la construcción de relaciones sólidas entre el museo y el visitante, para administrar las operaciones diarias con un sistema de gestión eficiente [8]. Otro software es Museum Space, disponible para ceso a través de la nube; es un software de gestión integral para museos, galerías, bibliotecas, archivos y lugares del patrimonio. Este sistema, contiene módulos para: gestión de la conservación, registro de imágenes, gestión de exposiciones, catalogación, gestión de préstamos, ubicaciones y movimientos, gestión de adquisiciones, gestión de bajas, entre otros [9].

En Colombia, se desarrolló el Prototipo de sistema de Recuperación de Información Artística de Las Exposiciones del Museo de Arte Moderno De Bogotá: Un análisis del Museo como Unidad le Información y la implementación de un Sistema de Información para recuperar las exposiciones del museo como un todo. El sistema permite recuperar la información que los guías brindan a los visitantes sobre el contexto de cada exposición. El sistema evita la pérdida de conocimiento dando un mayor sentido al trabajo de todas las personas que producen información en el Museo de Arte Moderno en Bogotá [10] 


\section{MATERIALES Y MÉTODOS}

Este trabajo se fundamenta en la información existente en la base de datos que actualmente posee el Museo, y en los criterios de los científicos que la manejan, sin llegar a niveles de detalle propios del objeto de estudio de cada uno de ellos. En cuanto la fundamentación técnica, se apoya en los principios de diseño de los SMBD [11], que permiten organizar la información,

Las fases del proyecto se presentan en la Tabla 1 y se describen a continuación:

Fase I: Mediante entrevistas a los científicos de Museo, desarrolladas inicialmente mediante entrevistas abiertas, y luego con entrevistas semidirectivas, se logra tener un conocimiento general del Museo y sus necesidades. En esta fase se aborda el análisis de los archivos de Excel en conde cada uno de ellos alberga una colección diferente. Su contenido está dividido en cuatro (4) hojas las cuales son: introducción, platilla, definiciones y vocabulario. Estos archivos se elaboraron de acuerdo a una clasificación entre zoología, dirigido a los animales, o botánica, dirigido a las plantas. A partir de ahí, los archivos se desarrollaron por subreinos y esto se hace tanto para las colecciones en exhibición como para las que están en bodega y de esta manera poderlas catalogar y relacionar fácilmente la llegada de especímenes nuevos. Las instrucciones acerca de cómo se debe hacer una platilla de documentación de registros biológicos está basada en los estándares de Darwin Core versión (2011-10-26) [12], donde menciona que son por lo general 159 elementos de utilidad a la hora de registrar un espécimen biológico, de los cuales solo cinco de ellos son obligatorios. Cada elemento consta de una columna y de un campo en específico. Como ejemplo de un archivo Excel de las colecciones, se presenta en la Tabla 1 un fragmento del archivo que registra los datos de la colección de ranas.

Tabla 16 Ejemplo del archivo en que se registran los datos de la colección de ranas

Ranos

occurrencell - basisofReco - institutionCo - collectionCo - catalogNumi - type D del registro $b$ Base del registn Código de la ins Código de la co Número de cat Tipo MLS:ran:1 Espécimen pres MLS MLS:ran:10 Espécimen pres MLS ran 1 Objeto físico - modified - language

MLS:ran:100 Espécimen pres MIS ran 10 100 Objeto fisico Modificado Idioma

MLS:ran:1000 Espécimen pres MLS ran Objeto fisico 2015-03-09 ES

MLS:ran:1001 Especimen pres MLS

$\operatorname{ran}$

ran Objeto fisico 15.03.09 ES 2015-05-11 ES

1000 Objeto fisico 2015-04-14 ES

1001 Objeto físico 2015-04-14 ES

De esta manera, el Museo de la Universidad de La Salle es fuente de estudio para la 
implementación de un Sistema de información, que tenga la capacidad de almacenar, modificar o integrar datos una manera eficaz, así como brindar información de acuerdo con los perfiles de usuario identificados en esta investigación. Que incluyen a los diferentes científicos que trabajan en el Museo, y a los visitantes, ya sean investigadores externos, estudiantes o solo visitantes [13]. Lo anterior permite organizar los elementos de manera que se conciba un modelo de datos y se desarrolle un vocabulario especializado para albergar el significado de los datos, lo que permite asegurar la calidad al almacenar los datos de forma organizada y conservar la correcta clasificación según la colección que corresponda, dando como resultado el modelo conceptual y el diccionario de datos. Como ejemplo, en la tabla 2 se presenta un fragmento del vocabulario propio de las colecciones del Museo.

Tabla 17 Vocabulario con las. palabras estratégicas que se deben utilizar a la hora de catalogar un espécimen.

\begin{tabular}{|c|c|c|}
\hline \multirow{7}{*}{ 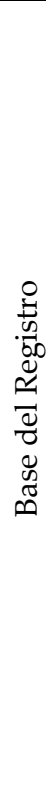 } & \multicolumn{2}{|c|}{ Registro biológico $\quad$ Evento } \\
\hline & Espécimen preservado & $\begin{array}{l}\text { El organismo esta preservado (muerto), vivió dentro de tiempos históricos y } \\
\text { existe (o alguna vez existió) una parte física que podría ser evaluada } \\
\text { nuevamente }\end{array}$ \\
\hline & Espécimen fosilizado & $\begin{array}{l}\text { El organismo vivió dentro de tiempos prehistóricos y existe (o alguna vez } \\
\text { existió) una parte física que podría ser evaluada nuevamente }\end{array}$ \\
\hline & Espécimen vivo & $\begin{array}{l}\text { Existe un espécimen disponible, se encuentra vivo (crece o metaboliza, no es } \\
\text { una parte dominante de un Espécimen preservado) al menos cuando se creó } \\
\text { el recurso }\end{array}$ \\
\hline & Observación humana & $\begin{array}{l}\text { El registro fue documentado sin recolectar evidencia física o digital que } \\
\text { pudiera ser evaluada nuevamente }\end{array}$ \\
\hline & Observación con maquina & $\begin{array}{l}\text { El registro fue documentado recolectando evidencia física o digital que } \\
\text { pudiera ser evaluada nuevamente. Fotografías, video o sonidos }\end{array}$ \\
\hline & $\begin{array}{l}\text { Lista de chequeo } \\
\text { nomenclatura }\end{array}$ & \\
\hline
\end{tabular}

Fase II: Sistematización de la información adquirida, con lo que mediante el diseñó lógico se obtiene como resultado el modelo de datos entidad relación, con la definición de atributos y sus respectivas restricciones, el cual debe validarse con los científicos del Museo. Como ejemplo en la figura 1 se presenta un fragmento del modelo entidad relación para albergar los datos de especímenes del reino anphibia, que entre otros alberga cecilias, ranas, sapos y salamandras. 
Relaciones

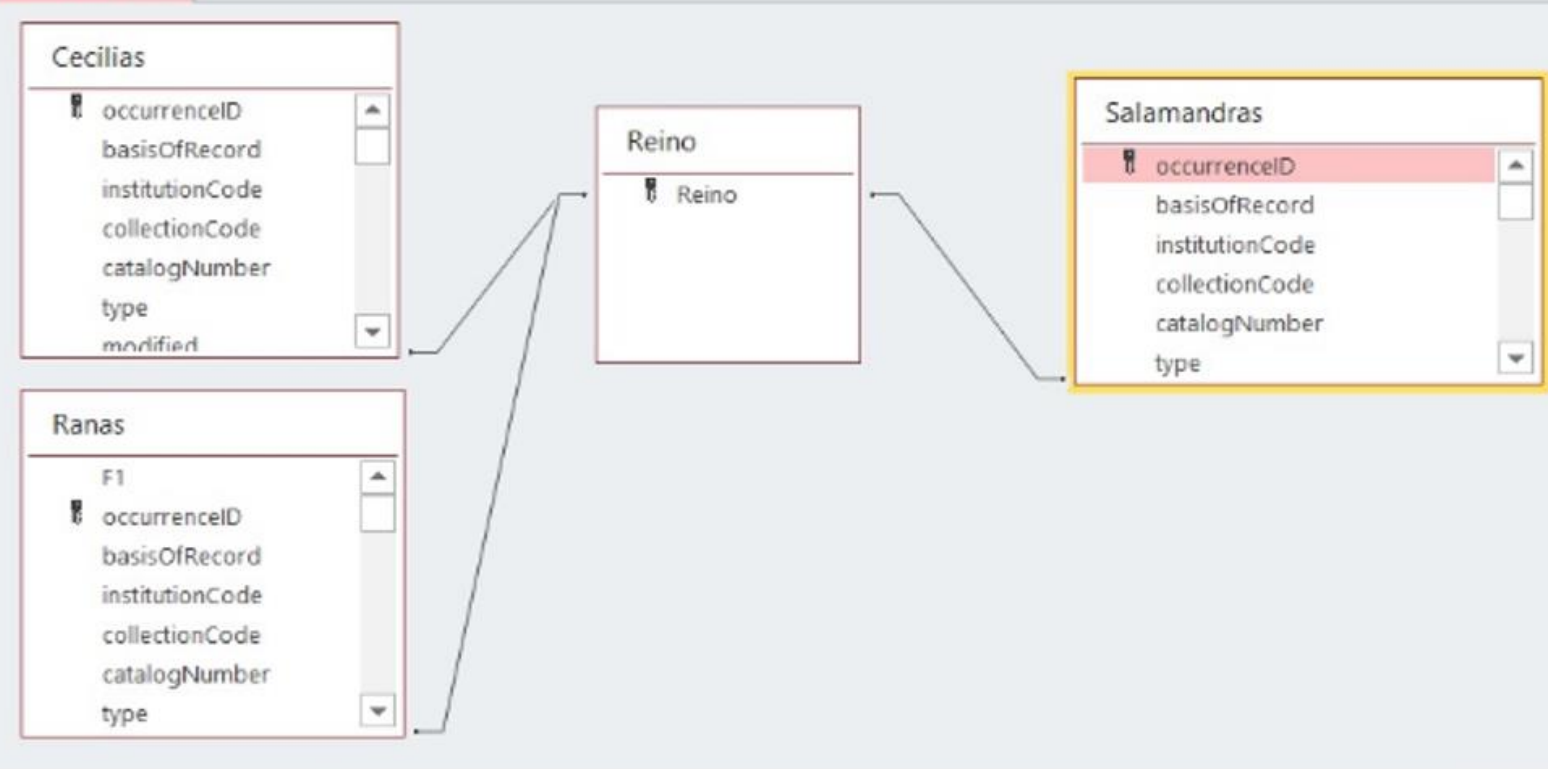

Figura 45 Fragmento de modelo entidad relación para el reino anphibia

Fase III: Implementar el modelo construyendo la base de datos, iniciando por importar los datos desde los archivos de Excel, Una vez que la base de datos reciba el contenido de los archivos de las colecciones, se verificará con los usuarios finales el almacenamiento de los registros en las nuevas tablas. Luego, se procederá a diseñar y programar los formularios y reportes, para lo cual se necesita identificar los diferentes usuarios y construir el documento de diseño de interfaces, con caso de uso y diagramas de secuencia, utilizando el modelado de sistemas de software con el lenguaje unificado de modelado (UML, por sus siglas en inglés, Unified Modeling Language). Luego, el documento de diseño se completará con la definición de la vista lógica de cada usuario. El resultado es la base de datos en Access con las tablas y vistas lógica, que aseguren que la información se disponga de acuerdo con el perfil de cada usuario mediante el desarrollo de formularios y reportes adecuados a cada uno de ellos.

Fase IV: Validar los avances obtenidos de la implementación del nuevo modelo con los usuarios del sistema, para lo cual se plantean dos ciclos de pruebas con diferentes actores. El primero para capacitar a los usuarios y validar el modelo desarrollado, y el segundo ciclo para verificar los ajustes que puedan requerirse como resultado del ciclo inicial de pruebas, de manera que el modelo pueda implementarse para su producción. 


\section{RESULTADOS Y ANÁLISIS DE RESULTADOS}

La información de las colecciones del Museo de la Universidad de la Salle requiere de un sistema de información soportado por una base de datos relacional, la cual se encuentra en construcción. Actualmente, este proyecto está su tercera fase; ya se desarrolló una primera fase del modelo, al obtener en la fase 1 los archivos de Excel que albergan los datos de las colecciones y poder plasmar las entidades y relaciones en el modelo conceptual para la nueva base de datos, así como definir el diccionario de datos para este sistema. Luego, en la fase dos, se construyó el modelo lógico y se definieron los atributos para cada tabla, con sus respectivas restricciones y reglas de validación, lo cual es fundamental para poder implementar el modelo en el motor Microsoft Access y diseñar las interfaces para cada uno de los roles asociados a los científicos, colaboradores y visitantes que puedan requerir información de alguna de las colecciones. En la definición de roles, también se establecieron los permisos para cada uno de ellos, dado que, para asegurar la calidad de la información, solo ciertos roles podrán almacenar, modificar o borrar datos de las tablas. Para cada uno de ellos se desarrollarán formularios, los cuales requieren programar reglas de validación, usando VBA, Visual Basic for Applications, por su sigla en inglés. De otra parte, todos los roles podrán generar reportes mediante consultas previamente definidas, las cuales están siendo desarrolladas en el lenguaje de consultas SQL. Tanto los formularios, como los reportes, van a requerir integrarse en una aplicación organizada, la cual se construirá usando VBA, lo que es fundamental a la hora de adquirir la información por cada usuario. De esta manera, este proyecto ayuda al Museo de la Universidad de la Salle a recolectar, procesar y disponer toda la información de cada uno de los especímenes que alberga de acuerdo con las colecciones correspondientes.

A lo largo del desarrollo del proyecto se han obtenido los siguientes avances:(1) Levantamiento de las políticas de manejo de datos en el Museo de acuerdo a sus necesidades. (2) Definición de los roles de usuario para el futuro sistema. (3) Construcción del diccionario de datos científico y técnico de acuerdo a la Norma Darwin Core. (4) Desarrolló de un primer módulo del sistema correspondiente a la colección de anfibios. Una vez verificado este módulo, la siguiente fase es agregar nuevos módulos para otras colecciones e integrar consultas entre los mismos.

\section{CONCLUSIONES}

La información con la que cuenta el Museo de la Universidad de la Salle esta almacenada de forma inapropiada mediante una serie de archivos Excel. Los datos se registran de acuerdo con la plantilla de documentación de registros biológicos, basada en los estándares de Darwin Core 
versión (2011-10-26) es de utilidad a la hora de registrar un espécimen biológico, lo que garantiza la calidad de los datos en cada archivo. El estándar Darwin Core debe ser respetado a la hora de implementar una nueva base de datos, para lo cual se escogió un modelo relacional, usando la metodología UML, el motor Microsoft Access y el lenguaje de programación VBA para la construcción del modelo. Además, el diseñó incluyó un análisis de seguridad de los datos mediante la identificación de perfiles de usuario a los cuales se les generaron las respectivas vistas lógicas sobre la nueva base de datos. De esta manera, se podrá administrar de manera más eficiente la información que maneja el Museo de La Salle en su día a día.

El nuevo modelo, se construye a partir del descubrimiento de las necesidades de los científicos del Museo integrando en un solo sistema todas las colecciones, adicionalmente el análisis del nuevo sistema incluye a los usuarios externos al Museo como son investigadores externos, estudiantes o visitantes ocasionales. Actualmente, el proyecto se encuentra en la tercera fase de implementación y se requiere que los científicos del Museo, como usuarios principales participen constantemente en la validación de cada una de las fases, implementando algunos de los principios de las metodologías agiles de desarrollo de software.

\section{REFERENCIAS}

[1] M. d. L. Salle, «Mision,» Universidad de La Salle, [En línea]. Available: http://museo.lasalle.edu.co/Museo-de-LaSalle/el-mls/detalle/mision. [Último acceso: 7 10 2020].

[2] Código de Deontología del ICOM para Museos, ICOM, 2017OM.

[3] Museo de La Salle, «Historia,» 27 2020. [En línea]. Available: http://museo.lasalle.edu.co/Museo-de-LaSalle/el-mls/detalle/historia/!

[4] «Acerca del Museo de La Salle,» Museo de La Salle, [En línea]. Available: http://museo.lasalle.edu.co/Museo-de-LaSalle/el-mls. [Último acceso: 105 2020].

[5] «Specify,» Specify, software, [En línea]. Available:

https://www.sustain.specifysoftware.org/. [Último acceso: 165 2020].

[6] I. NetXposure, «netx,» NetXposure, Inc, 2019. [En línea]. Available: https://www.netx.net/. [Último acceso: 0909 2020].

[7] Lucidea, «Argus,» Lucidea, 2020. [En línea].
Available: https://lucidea.com/argus/. [Último acceso: 0909 2020].

[8] I. Blackbaud, «Altru,» Blackbaud, Inc, 2020. [En línea].

Available: https://www.blackbaud.com/products/blackba ud-altru. [Último acceso: 0909 2020].

[9] S. Group, «Museum space,» Sirma Group, 2018. [En línea]. Available: https://museumspace.com/. [Último acceso: 09 09 2020].

[10] M. F. HINESTROSA, «PROTOTIPO DEL SISTEMA DE INFORMACIÓN DE RECUPERACIÓN DE,» Bogotá, 2009.

[11] M. Mannino, Administración de Bases De Datos, Diseño y Desarrollo de aplicaiones, Mexico: McGraw Hill, 2011.

[12] Instituto de Investigación de Recursos Biológicos Alexander von Humboldt. , «Perfil estándar Darwin Core (Adaptación IAvH). Infraestructura Institucional de Datos, Bogotá D.C., Colombia,» 2016. [En línea]. Available: https://sites.google.com/humboldt.org.co/i2dw iki/perfil-est $\%$ C3\%A1ndar-darwin-core. 
[Último acceso: 194 2020].

[13] K. L. J. Laudon, Systemas de Información, New
York: Pearson, 2015. 